\begin{abstract}
Forensic gait analysis is the analysis, comparison and evaluation of features of gait to assist in the investigation of crime. Analysis refers to the process of observing features of gait and assessing their reliability for use in comparison, within the limitations of the quality of the footage. Forensic gait analysis often uses closed circuit television (CCTV) cameras to view the figure of interest. A limitation associated with using CCTV cameras is the viewing angle provided of the figure of interest. The aim of this study was to test the effect of viewing angle on observations made about the orientation of a figure's feet whilst the figure was walking. The results will supplement the forensic gait analyst's understanding of uncertainty measures. An opportunistic sample of 31 participants, with some knowledge of observational gait analysis, was recruited. Each participant viewed nine clips of an Avatar walking with its feet abducted to 30 degrees. Viewing angles ranged from 0 degrees to 180 degrees in 22.5 degree increments from the Avatar's left side. Participants were asked to comment on the orientation of both the left and right feet. The most reliable viewing angle to make judgements about the orientation of the feet was in the frontal plane, from the rear of the avatar. The most unreliable viewing angle was obliquely, from the front and side of the avatar. More incorrect judgements were made about the foot situated furthest from the camera, than the foot closest to it. Viewing angle relative to the figure being observed can influence judgements about angle of gait, and practitioners should be mindful of this when analysing, comparing and evaluating this feature as part of forensic gait analysis.
\end{abstract}

\title{
The effect of viewing angle on observations of foot orientation in forensic gait analysis
}

\section{Introduction}

Forensic gait analysis has been defined as the "analysis, comparison and evaluation of features of gait to assist in the investigation of crime" [1]. Forensic gait analysis can assist criminal investigations because despite the absence of any current evidence to support the assertion that gait is unique [2], features of gait can be highly discriminatory, particularly when used in combination [3, 4]. By analysing, comparing and evaluating these features, forensic gait analysis can assist the process of identification [3] rather than identifying an individual in an open population [1].

Factors exist that may influence the practitioner's ability to reliably analyse features of gait. 'Analysis' refers to the process of observing features of gait and assessing their reliability for use in comparison within the limitations of the quality of the footage [1]. The limitations or factors that can introduce error and uncertainty have been identified in earlier research [3,5]. Birch et al. [5] developed that enables practitioners to assess the quality of footage by considering the impact of each factor on their observations whilst following a standardised process. Key factors listed by Birch et al. include 'picture', 'lighting', 'direction', 'frame rate' and 'subject' (this can include the effects of clothing and number of steps taken). 'Direction' as described by Birch et al. [5] refers to the position of the camera relative to the figure being recorded and observed. This category, like the majority listed, is displayed as a continuum with 'directly from the side' and 'directly from the front or back' forming each extreme. The Forensic Gait Analysis Primer for Courts [2] recognised that a tool existed for assessing footage for use in forensic gait analysis, but stated there was little detail given on 'where on the continuum optimum footage would sit'. Whilst forensic gait analysts take viewing angle into consideration and its potential influence on their observations, there is no known published data on the level of uncertainty associated with this factor [2]. The Forensic Science Regulator requires forensic units in the UK that perform qualitative methods to subject these methods to the same level of evaluation as 
required for quantitative tests [6]. As the results reported by forensic gait analysts rely on qualitative methods that have associated uncertainties, the margin of uncertainty needs to be better understood to reduce the potential impact upon their reported findings [6].

Research conducted by Birch et al. [7 \& 4] demonstrated that analysts were more likely to make correct identifications of a suspect walker from a sample of target walkers when the walkers were viewed in the same plane. When the planes were different for the target and suspect walkers, views of the walker from their front yielded more correct responses, whereas most errors occurred when the walker was viewed from the back. However, this finding was secondary to the main aim of their study, which was to investigate the effectiveness of visual gait analysis as a means of identification. Participants used a combination of features of gait to recognise the target walker from the suspect walkers. Furthermore, they could only use features for comparison that were both demonstrated by the walker and also visible in the particular plane under examination. As this research did not specifically test the participants' ability to observe a single feature of gait using various viewing angles, it did not control for other potential influencing factors. Larson et al. [8] reported that the optimum viewing angle for observing features of gait was in the frontal plane. However, they acknowledge that this finding resulted from case work experience as opposed to systematic investigation.

This study investigates whether viewing angle can influence our ability to make accurate observations about angle of gait. Whilst it is recognised that the outcome will not be true for all features of gait, particularly those demonstrated in different planes from that under investigation, the results are intended to add to the evidence base available to forensic gait analysts and those training in the field.

\section{Materials and Method}

An opportunistic sample of 31 participants was recruited from the student population of a university in the UK. Ethical approval for the study was obtained from the Faculty Ethics Committee at the study university. Participants were fully informed of the study and each participant gave their consent to participate. Participants included in the study were over 18 years of age and had some knowledge of observational gait analysis. Individuals with known visual impairments or those with health conditions affected by moving or flashing images were not permitted to take part.

\subsection{Data collection}

A series of video clips were produced showing a computer-generated person (avatar) walking. The walking pattern was constructed with the aid of motion capture of a human adult walking. Any asymmetry or idiosyncrasy of gait was removed in order to obtain a completely common gait pattern. Make human, an open source program, was used to create the avatar based on the motion capture [9]. The open source program Blender was used to create an environment and to create the 3D clips [10]. The frame rate in the clips was set to 30 frames per second (FPS) and the resolution of the created animations was $960 \times 540$ pixels. Although resolution and frame rate in real closed circuit television (CCTV) footage varies considerably $[3,11]$, they are on average comparable to the settings used in this study.

The use of a computer-generated avatar allowed features of gait to be 'fixed' which may otherwise show slight variation in a human walker. The feature of gait selected for analysis in this study was angle of gait; the extent to which the feet point inwards or outwards from the line of progression. This feature is commonly analysed as part of forensic gait analysis $[3,8,12-13)$ due to its discriminatory potential [14]. Therefore, it is a feature that participants with some knowledge of gait analysis can recognise. Both of the avatar's feet were turned out by $30^{\circ}$. 'Normal' angle of gait values for adults vary as demonstrated by the literature. Reported ranges for healthy adults include $-9.7^{\circ}$ (in-toeing) to $14.3^{\circ}$ (out-toeing) [15] and $6^{\circ}$ to $21^{\circ}[16]$ with mean values of $13^{\circ}[16]$ and $4.5^{\circ}[15]$ for males and $1.4^{\circ}$ 
for females [15]. Therefore, turning the feet out by $30^{\circ}$ represents a marked, abducted foot position. This provided a clearly discernible, unambiguous feature that would enable any effect observed to be attributed to viewing angle. All other features of gait were held constant between clips.

A number of considerations were taken into account when selecting which viewing angles to investigate. A sufficient number of clips were required to test the difference between viewing angles whilst avoiding the effects of participant fatigue or learning on the participants' judgement. Yu et al. [17] used 11 clips of footage that differed by $18^{\circ}$ to give a range of viewing angles from $0^{\circ}$ to $180^{\circ}$ of the walker's left side. However, by using $18^{\circ}$ increments, a view showing equal amounts of the walker's front and side or back and side, at $45^{\circ}$ and $135^{\circ}$ respectively, are not available. Therefore, this study used increments of $22.5^{\circ}$ over a range of $180^{\circ}$ to produce 9 clips of footage of the avatar's left side. This gave views in the frontal plane of the avatar's front $\left(0^{\circ}\right)$ and back $\left(180^{\circ}\right)$, a view in the sagittal plane of the avatar's left side $\left(90^{\circ}\right)$, three oblique views from the avatar's front $\left(22.5^{\circ}, 45^{\circ}\right.$, $\left.67.5^{\circ}\right)$ and three oblique views from the avatar's back $\left(112.5^{\circ}, 135^{\circ}, 157.5^{\circ}\right)$ (Table 1$)$. 
Table 1. Viewing angles for each clip with associated viewing order

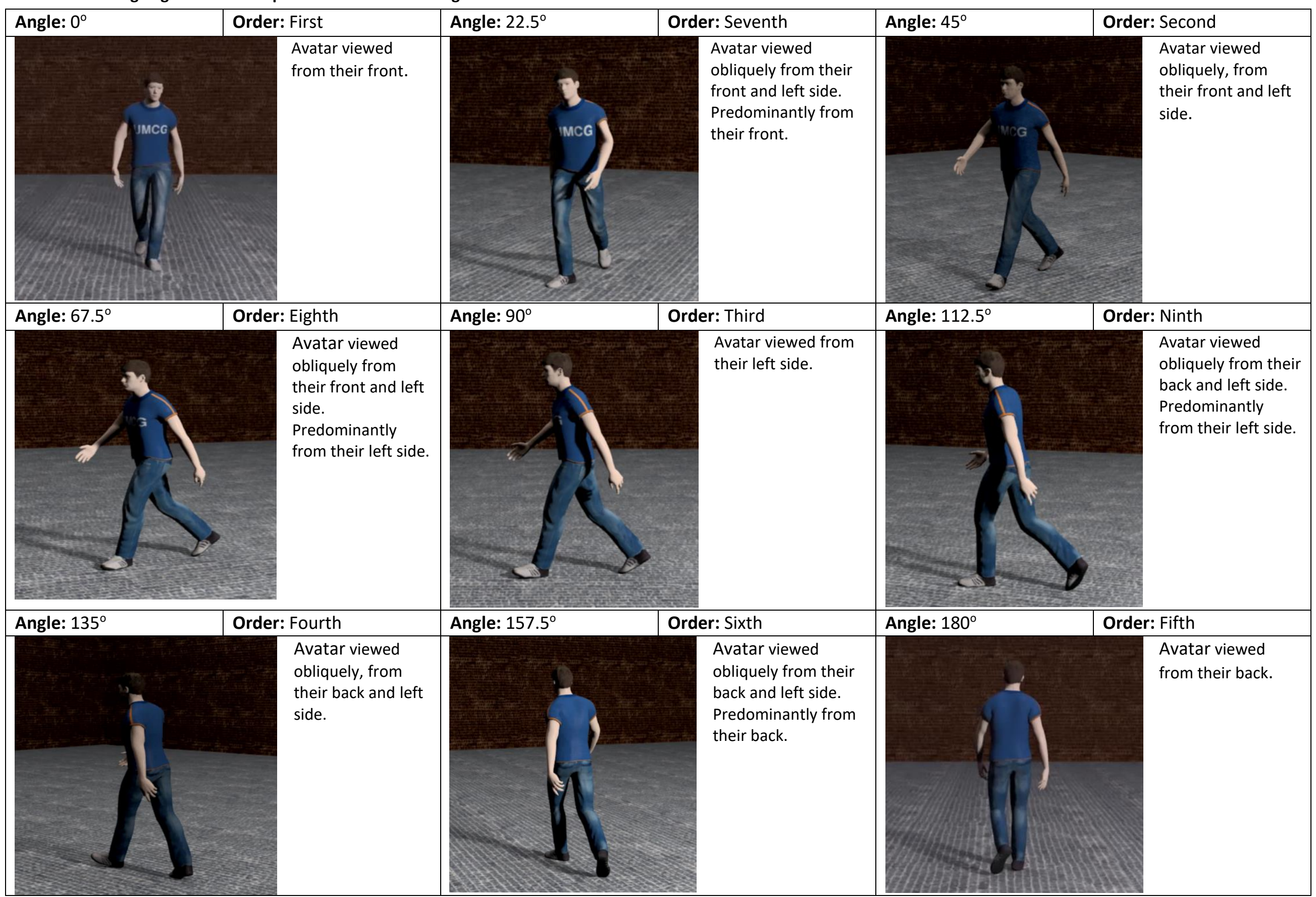


All participants viewed each of the nine clips of footage in a randomised order (Table 1) using a PowerPoint slide show. For each clip, the participants were required to make a decision as to whether they considered the figure's left and right foot to be either pointing markedly outwards, pointing slightly outwards, pointing forwards, pointing slightly inwards or pointing markedly inwards during the stance phase of gait. Participants also had an option to mark the foot position as 'not discernible' if, in their opinion, the viewing angle did not provide a sufficient view of the feet. The participants were required to make one judgement for each foot and for each clip. This produced 18 judgements for each participant. The participants were aware that they were viewing the same figure in each of the nine clips, but were not aware that the angle of gait was held constant in each clip. At $0^{\circ}$ and $180^{\circ}$,

both left and right feet were an equal distance from the camera. However, for camera angles from $22.5^{\circ}$ to $157.5^{\circ}$, the left foot was closer to the camera than the avatar's right foot. Each participant was provided with a data collection sheet to record their observations. Training was not provided. No other data was collected on participants.

\subsection{Statistical Methods}

The sample was summarised descriptively. A repeated measures analysis of variance (RM-ANOVA) was conducted on the data to assess the significance of differences in correctness of responses elicited from different viewing angles. Significant differences between individual viewing angles were assessed using Bonferroni-corrected pairwise comparisons. Due to the angular nature of measurements, it was not appropriate to model the effect of angle with a linear trend. Paired samples $t$-testing was conducted on the data to assess the significance of differences in correctness of responses elicited from the left (near camera) or right (distant to camera) foot.

Each judgement made by the participants was influenced to a varying degree by the participant making the judgement, the viewing angle and the foot being assessed. The relative contribution of each of these elements on the judgements made required assessment. This was achieved by formulating the data as a hierarchical, multilevel data structure; with participants forming the higher level of the model (level 3), and the repeated measures recorded from these participants forming the middle (level 2) and lower levels (level 1). The angle of view corresponded to the middle level and the foot being assessed formed the lower level of the model. As camera views at $0^{\circ}$ and $180^{\circ}$ showed both left and right feet at an equal distance from the camera, data from these camera angles was not considered for this analysis. Only data from camera angles between $22.5^{\circ}$ and $157.5^{\circ}$ inclusive were used. A logistic multilevel model was conducted with the dichotomous outcome of correctness of response measured at the lowest level of the analysis. Level-2 and Level-3 residual variances were obtained directly from the multilevel model. Level-1 variance was estimated using a simulation method: in binary and other discrete response multilevel models, there is no single variance partition coefficients (VPC) measure since the Level-1 variance is a function of the mean, which depends on the values of the explanatory variables in the model. Therefore, the VPC itself depends on any explanatory variables. In the current context, as no participant-level variables were available, null models were used in the simulation to estimate the Level-1 variance. The VPCs corresponding to each level of the model were determined from the individual level variances.

\section{Results}

\subsection{Descriptive Statistics}

All data was removed for one participant who made multiple errors on their data collection sheet. One set of data was voided for a participant who made one error on their data collection sheet when viewing the avatar at $22.5^{\circ}$. Errors for both participants involved inadvertently marking multiple 
responses for one foot, rather than one response as instructed. Therefore, a total of 538 judgements were produced, 269 for each foot. 29 participants made 18 judgements each ( 9 for the left foot, 9 for the right foot) and one participant made 16 judgements ( 8 for each foot). 8 of the 9 clips of footage yielded 60 judgements; one clip $\left(22.5^{\circ}\right)$ produced 58 judgements for analysis (Table 2).

Table 2 Participant responses for each angle of view

\begin{tabular}{|c|c|c|c|c|c|c|c|c|c|c|c|c|c|}
\hline \multirow[t]{2}{*}{$\begin{array}{l}\text { Clip \& } \\
\text { Viewing } \\
\text { Angle }\end{array}$} & \multicolumn{2}{|c|}{$\begin{array}{l}\text { Pointing } \\
\text { Markedly } \\
\text { Outwards }\end{array}$} & \multicolumn{2}{|c|}{$\begin{array}{l}\text { Pointing } \\
\text { Slightly } \\
\text { Outwards }\end{array}$} & \multicolumn{2}{|c|}{$\begin{array}{l}\text { Pointing } \\
\text { Forwards }\end{array}$} & \multicolumn{2}{|c|}{$\begin{array}{l}\text { Pointing } \\
\text { Slightly } \\
\text { Inwards }\end{array}$} & \multicolumn{2}{|c|}{$\begin{array}{l}\text { Pointing } \\
\text { Markedly } \\
\text { Inwards }\end{array}$} & \multicolumn{2}{|c|}{$\begin{array}{l}\text { Not } \\
\text { Discernible }\end{array}$} & \multirow[t]{2}{*}{$\begin{array}{l}\text { Total } \\
\text { Responses }\end{array}$} \\
\hline & Left & Right & Left & Right & Left & Right & Left & Right & Left & Right & Left & Right & \\
\hline $1\left(0^{\circ}\right)$ & 7 & 8 & 20 & 22 & 3 & 0 & 0 & 0 & 0 & 0 & 0 & 0 & 60 \\
\hline $2\left(45^{\circ}\right)$ & 19 & 2 & 10 & 10 & 1 & 15 & 0 & 2 & 0 & 0 & 0 & 1 & 60 \\
\hline $3\left(90^{\circ}\right)$ & 10 & 7 & 18 & 14 & 2 & 6 & 0 & 1 & 0 & 0 & 0 & 2 & 60 \\
\hline $4\left(135^{\circ}\right)$ & 11 & 15 & 18 & 14 & 1 & 0 & 0 & 0 & 0 & 1 & 0 & 0 & 60 \\
\hline $5\left(180^{\circ}\right)$ & 10 & 18 & 20 & 12 & 0 & 0 & 0 & 0 & 0 & 0 & 0 & 0 & 60 \\
\hline $6\left(157.5^{\circ}\right)$ & 8 & 21 & 20 & 8 & 2 & 0 & 0 & 0 & 0 & 0 & 0 & 1 & 60 \\
\hline $7\left(22.5^{\circ}\right)$ & 11 & 2 & 16 & 13 & 2 & 13 & 0 & 0 & 0 & 0 & 0 & 1 & 58 \\
\hline $8\left(67.5^{\circ}\right)$ & 13 & 3 & 15 & 7 & 2 & 19 & 0 & 1 & 0 & 0 & 0 & 0 & 60 \\
\hline $9\left(112.5^{\circ}\right)$ & 8 & 18 & 18 & 10 & 4 & 1 & 0 & 0 & 0 & 0 & 0 & 1 & 60 \\
\hline Total & 97 & 94 & 155 & 110 & 17 & 54 & 0 & 4 & 0 & 1 & 0 & 6 & 538 \\
\hline
\end{tabular}

For the purposes of analysis, markedly pointing outwards and slightly pointing outwards were combined to form the category 'pointing outwards'. Markedly pointing inwards and slightly pointing inwards were combined to form the category 'pointing inwards' (Table 3). Whilst the literature [1516] and the authors of this study consider $30^{\circ}$ to be markedly pointing out, the raw data in this study suggested that most participants perceived the foot orientation of the avatar to be slightly abducted (Table 2). Whilst participants had some knowledge of gait analysis, they were not considered to be experts in the field and training was not provided to demonstrate what a 'markedly' or 'slightly' pointing in or out foot position looked like. Therefore, these categories were expanded to account for this potential limitation.

Table 3 Participant responses for each angle of view with combined foot orientation data

\begin{tabular}{|c|c|c|c|c|c|c|c|c|c|}
\hline \multirow[t]{2}{*}{$\begin{array}{l}\text { Clip \& } \\
\text { Viewing Angle }\end{array}$} & \multicolumn{2}{|c|}{$\begin{array}{l}\text { Pointing } \\
\text { Outwards } \\
\end{array}$} & \multicolumn{2}{|c|}{$\begin{array}{l}\text { Pointing } \\
\text { Forwards }\end{array}$} & \multicolumn{2}{|c|}{$\begin{array}{l}\text { Pointing } \\
\text { Inwards }\end{array}$} & \multicolumn{2}{|c|}{$\begin{array}{l}\text { Not } \\
\text { Discernible }\end{array}$} & \multirow[t]{2}{*}{$\begin{array}{l}\text { Total } \\
\text { Responses }\end{array}$} \\
\hline & Left & Right & Left & Right & Left & Right & Left & Right & \\
\hline $1\left(0^{\circ}\right)$ & 27 & 30 & 3 & 0 & 0 & 0 & 0 & 0 & 60 \\
\hline $2\left(45^{\circ}\right)$ & 29 & 12 & 1 & 15 & 0 & 2 & 0 & 1 & 60 \\
\hline $3\left(90^{\circ}\right)$ & 28 & 21 & 2 & 6 & 0 & 1 & 0 & 2 & 60 \\
\hline $4\left(135^{\circ}\right)$ & 29 & 29 & 1 & 0 & 0 & 1 & 0 & 0 & 60 \\
\hline $5\left(180^{\circ}\right)$ & 30 & 30 & 0 & 0 & 0 & 0 & 0 & 0 & 60 \\
\hline $6\left(157.5^{\circ}\right)$ & 28 & 29 & 2 & 0 & 0 & 0 & 0 & 1 & 60 \\
\hline $7\left(22.5^{\circ}\right)$ & 27 & 15 & 2 & 13 & 0 & 0 & 0 & 1 & 58 \\
\hline $8\left(67.5^{\circ}\right)$ & 28 & 10 & 2 & 19 & 0 & 1 & 0 & 0 & 60 \\
\hline $9\left(112.5^{\circ}\right)$ & 26 & 28 & 4 & 1 & 0 & 0 & 0 & 1 & 60 \\
\hline Total & 252 & 204 & 17 & 54 & 0 & 5 & 0 & 6 & 538 \\
\hline
\end{tabular}

The only viewing angle that produced $100 \%$ of correct judgements for both feet was $180^{\circ}$ and for the right foot $0^{\circ}$ (Table $2 \& 3$ ). Both viewing angles were in the frontal plane where the avatar was viewed from its back and front respectively. In the remaining clips, more correct judgements were made 
about the foot closer to the position of the camera (left) than that furthest away (right). The left foot was considered to be discernible by all participants whereas in 6 out of 269 judgements, the right foot was considered not to be discernible (Table $2 \& 3$ ). Such cases were considered to be equivalent to an incorrect response in this study because the 'ground truth' of the angle of gait was known. In casework, an 'indiscernible' response can be a necessary and accurate observation, particularly where a feature cannot reliably be described. In practice, many factors are likely to have contributed to a feature being indiscernible. However in this study, the only variable being manipulated was viewing angle, and as such, whether or not the feet could be seen resulted from this change.

All nine clips yielded a high number of correct responses for the left foot with a viewing angle of $112.5^{\circ}$ showing the lowest score (86.6\%). When errors were made regarding left foot position, participants perceived the foot to be pointing forwards $(6.7 \%)$. They did not perceive it to be pointing inwards in any of the clips. However, participants considered the right foot to be pointing forwards in $20 \%$ of cases and in $2 \%$ of cases, participants considered it to be pointing inwards (Table 3 ). For the right foot, $0^{\circ}$ and angles between $112.5^{\circ}-180^{\circ}$ (an oblique view, from the back and side) produced the most correct responses. Most incorrect judgements for the right foot were made when viewing angles ranged between 22.5 and $67.5^{\circ}$ (Table 3). Collectively, these viewing angles show the figure obliquely, from their front-left side.

\subsection{Statistical Results}

The mean number of correct responses given to the 18 judgements made by each participant was 15.2 (SD 1.86); with a range of 12 to 18 correct. This represented an average of $84.4 \%$ of correct responses per participant. Missing data amounted to two values from one participant (0.4\%). Missing data was not imputed.

Some substantial variation in correctness of response with angle of view was observed. Combining responses relating to the left and right foot, the angle of view associated with the largest number of correct responses was $180^{\circ}$, in which 30 out of 30 responses $(100.0 \%)$ were judged to be correct. The angle of view associated with the least number of correct responses was $67.5^{\circ}$, in which 19 out of 30 responses (63.3\%) were judged to be correct. In general, a very low angle $\left(0^{\circ}\right)$ and angles greater than $90^{\circ}$ were associated with greater numbers of correct responses, recorded in Figure 1. Variation between participants was very low: $95 \%$ confidence intervals (not shown) correspond to a margin of error of less than \pm 0.2 in all cases. The high values recorded at both extremes of the distribution reflect the circular nature of the measure.

Figure 1: Percentage of correct responses associated with each angle of view 


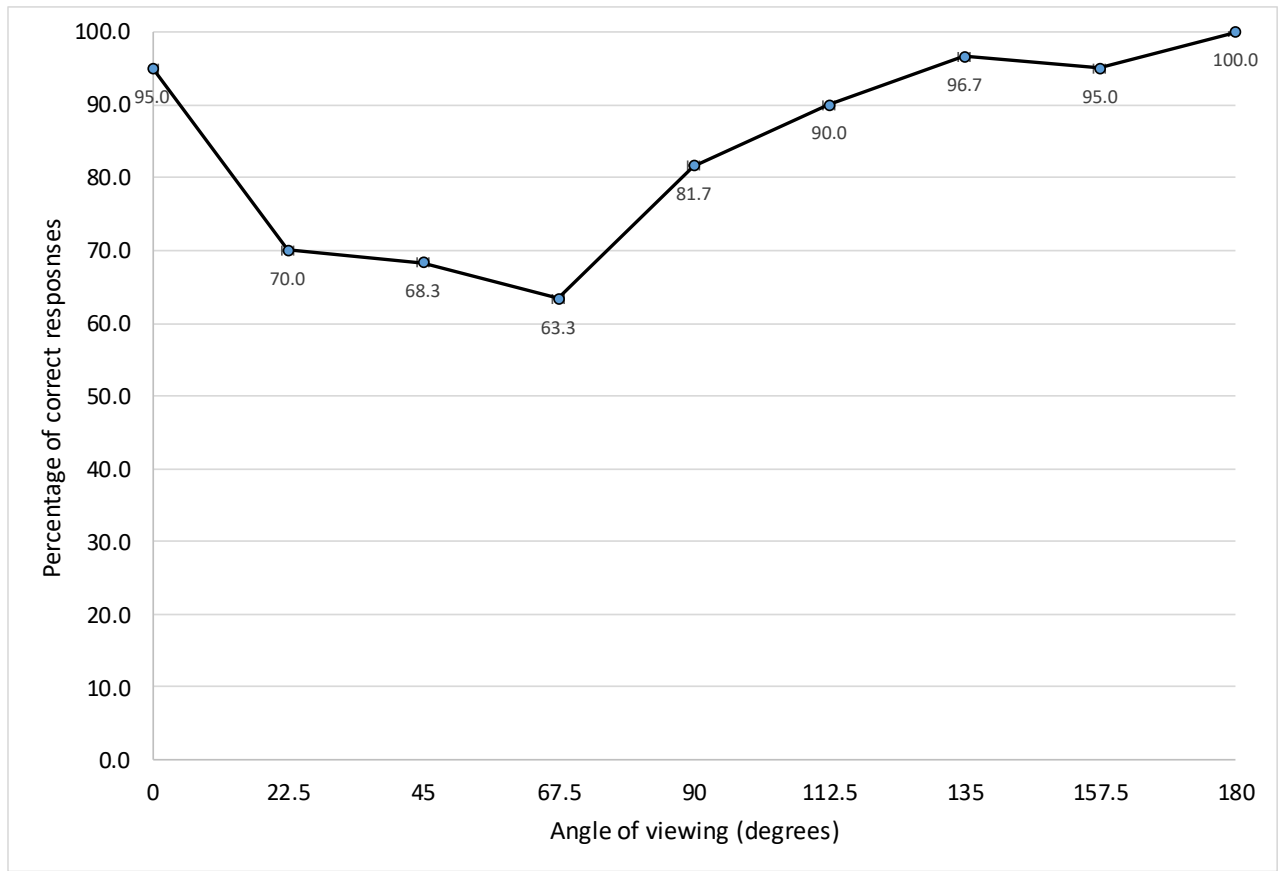

A repeated measures analysis of variance (RM-ANOVA) using the Greenhouse-Geisser correction to account for violation of the sphericity assumption revealed the number of correct scores to be significantly different between angles of view $\left(F_{5.15,232}=13.8 ; p<0.001\right)$. The effect was moderate in magnitude by the partial-eta squared statistic (partial- $\eta^{2}=0.322$ ). Bonferroni-corrected pairwise comparisons revealed the following significant differences in scores:

- Correctness level measured at $0^{\circ}$ significantly lower than correctness levels measured at: $22.5^{\circ}(p=0.006) ; 45^{\circ}(p=0.001) ; 67.5^{\circ}(p=0.001)$

- Correctness level measured at $22.5^{\circ}$ significantly lower than correctness levels measured at: $135^{\circ}(p=0.002) ; 157.5^{\circ}(p=0.014) ; 180^{\circ}(p<0.001)$

- Correctness level measured at $45^{\circ}$ significantly lower than correctness levels measured at: $112.5^{\circ}(p=0.025) ; 135^{\circ}(p<0.001) ; 157.5^{\circ}(p<0.001) ; 180^{\circ}(p<0.001)$

- Correctness level measured at $67.5^{\circ}$ significantly lower than correctness levels measured at: $90^{\circ}(p=0.041) ; 112.5^{\circ}(p=0.007) ; 135^{\circ}(p<0.001) ; 157.5^{\circ}(p<0.001) ; 180^{\circ}(p<0.001)$

- Correctness level measured at $90^{\circ}$ significantly lower than correctness levels measured at: $180^{\circ}(p=0.041)$.

\subsubsection{Variation between near and far foot assessments}

Of the 269 assessments associated with the left (near) foot, 252 (93.7\%) were correct. Of the 269 assessments associated with the right (far) foot, 204 (75.8\%) were correct. The mean percentage of correct responses per participant was 93.3\% (SD 9.77\%) for the left foot assessments and $75.6 \%$ (SD $17.3 \%$ ) for the right foot assessments; hence a difference in mean of $17.8 \%$ (Figure 2).

Figure 2: Correct responses associated with each foot assessment 


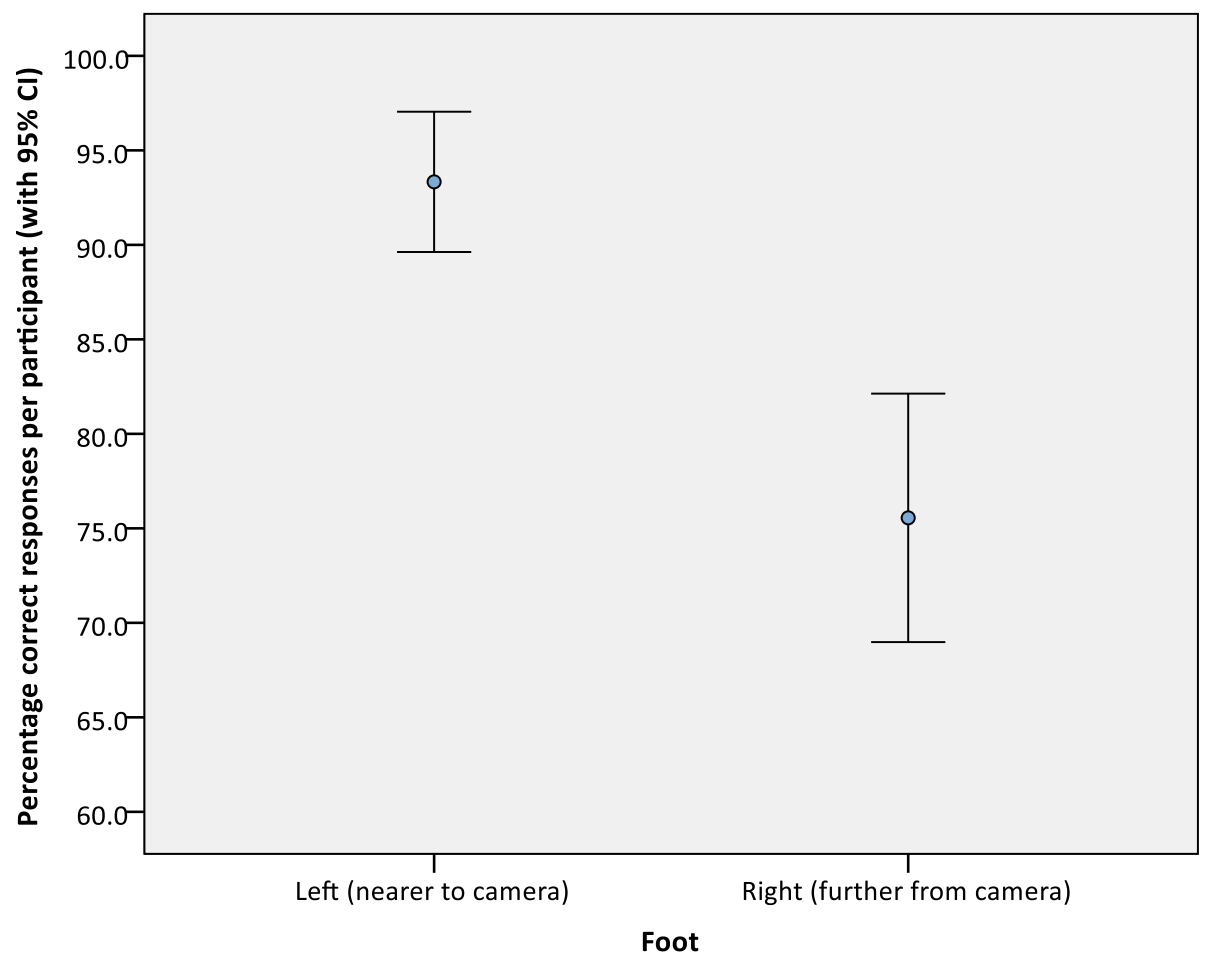

A paired samples $t$-test revealed this difference to be statistically significant at the $5 \%$ significance level $\left(t_{29}=5.00, p<0.001\right)$. A 95\% confidence interval for the difference was given by $(0.945,2.25)$.

\subsubsection{Variance components analysis}

The multilevel logistic model used to assess the variance components associated with each factor is:

$\ln \frac{\pi_{i j k}}{1-\pi_{i j k}}=1.458+u_{0 j k}+v_{0 k}$

where $u_{0 j k}$ and $v_{j k}$ are the variances at the participant and angle-level respectively;

with $u_{0 j k} \sim N(0,6.22)$ and $v_{j k} \sim N(0,0.312)$

Using the simulation method, the distribution of the foot-level variance $w_{i j k}$ is given by $w_{i j k} \sim N(0,0.150)$. This corresponds to variance partition coefficients of $1.73 \%$ at Level- $1,93.6 \%$ at Level-2 and $4.7 \%$ at Level-3; which may be considered to be error variance.

Hence, the majority of variance in responses arose from changes in the angle of view; withinparticipant variance (error variance) was very low. Variation due to angle of incidence amounted to $98.2 \%$ of variance amongst controllable error sources.

\section{Discussion}

The aim of this study was to investigate whether viewing angle could significantly influence how angle of gait was observed. This is considered particularly important in forensic gait analysis when considering the position of CCTV cameras relative to the figure of interest. The results of this study showed that whilst viewing angle was initially considered to be the only variable under investigation, two variables were capable of influencing judgements: viewing angle and the position of the feet relative to the camera. 
The results showed that overall, the level of accuracy exhibited by participants was very high (84.4\%). Where inaccurate judgements occurred, they could be attributed to changes in viewing angle and/or whether the near or far foot was being assessed, because participant responses exhibit a high degree of self-similarity.

Most participants varied their responses depending on the angle of view. The critical region appeared to be $22.5^{\circ}$ to $67.5^{\circ}$, an oblique angle from the front and side of the figure being observed. Over this region, responses were less accurate than in other viewing angles. Levels of correctness in favourable angles were up to $58 \%$ higher than in unfavourable angles.

Excluding analyses derived from angles at which both feet were equal distances from the camera, participants gave more correct judgements when observing the foot closer to the camera $(93.7 \%$ correct) than the more distant foot ( $75.8 \%$ correct). Levels of correctness vary between left (near) and right (far) feet to a lesser extent than between viewing angles. Levels of correctness for the near foot were about $24 \%$ higher than for the far foot. Therefore, angle of view influenced judgements made by participants more than whether the foot was closer or further from the camera. Approximately $98 \%$ of controllable variation is sourced from angle of view.

The results of this study suggest that the least reliable viewing angles to make judgements about angle of gait are between $22.5^{\circ}$ and $67.5^{\circ}$, particularly when observing the foot furthest from the camera. Observing a walker from their back in the frontal plane $\left(180^{\circ}\right)$ was the most reliable viewing angle for assessing angle of gait. However, forensic gait analysts must be mindful not to use these viewing angles as absolute figures, but as guidance. Firstly, viewing angles were increased in increments of $22.5^{\circ}$ to form defined categories, but angle of view is a continuum of $360^{\circ}$. Therefore, the angles forming the extremes of these categories do not represent the exact point at which a viewing angle becomes more or less reliable. Secondly, in live casework the viewing angle relative to the figure of interest is not known. Therefore, the forensic practitioner should apply the qualitative findings derived from this research to assist their practice rather than using the quantitative figures. For example, practitioners should employ caution when forming an opinion about the position of the feet, particularly the foot furthest from the camera, when the figure of interest is viewed obliquely, from the front and side. If additional footage is available from an alternative viewing angle, the practitioner should refer to this prior to forming their opinion. If additional footage is not available, the practitioner must be mindful of this margin of uncertainty when forming their final conclusions and they should document the limitations in their report. However, these results should not prevent forensic gait analysts from assessing angle of gait using frontal-oblique viewing angles, as the overall results of this study showed high levels of accurate judgements. The research should be used to supplement the skill and experience of the practitioner by providing an evidence base to support their decision making process.

93.7\% of judgements made about the position of the foot closest to the camera were correct. Whilst these results may increase confidence in judgements made about the foot nearest the camera, practitioners must be aware that the foot angle used in this research was markedly abducted $\left(30^{\circ}\right)$, and therefore more easily identifiable. Practitioners must also recognise if other limitations (in addition the viewing angle) are present in the footage, this may reduce their level of confidence.

The findings reported in this research differ to those described by Birch et al. [4, 7]. In 2013, Birch et al. [7] reported that the most useful view to make gait observations was from the side of the walker. However, in their 2020 study, Birch et al. [4] concluded that for experienced analysts, the most reliable view was from the front of the walker and for novices, it was an oblique posterior view of the walker. The most unreliable viewing angle for both experienced and novice analysts was the posterior view [4]. However, these studies were not investigating which viewing angle was the most appropriate for observing features of gait, which may account for some of these differences. Furthermore, it is likely 
that the participants in these studies used a number of features of gait for the purpose of recognition. Birch et al. [7] stated that most participants used upper body features (such as arm swing) to make their decisions. Therefore, the most useful viewing angles reported by Birch et al. [7, 4] may differ to the present study because different features were being observed by the participants and these different features may be better observed in different planes.

Supporting the findings of this study were results published in 2019 by Birch et al. [12]. They used a clip of footage showing an oblique, front-left view of an avatar walking to investigate the repeatability and reliability of the Sheffield Features of Gait Tool. A feature listed in this tool is the position of the feet in the stance phase of gait. Birch et al. [12] noted that one of the lowest scoring sections (in addition to others) included the position of the feet during the stance and swing phase of gait. This oblique, front-left perspective was similar to that used in this study that yielded the most unreliable results. This may provide support for the assertion that an oblique, front-side view of a figure may be the most unreliable when making judgements about angle of gait. However, in their 2013 and 2020 research, Birch et al. [7 \& 4] used human walkers, whereas in this study and that published by Birch et al. in 2019 [12], avatar walkers were used. Whilst further research may be required to establish whether the use of an avatar has an effect on observations made, a case report by Larson et al. [8] supports the notion that frontal plane footage is the preferred viewing angle for observing angle of gait. Furthermore, research by Jokisch et al. [18] found that participants made more correct judgements (using multiple features of gait) using frontal plane as opposed to viewing angles of $30^{\circ}$ and $90^{\circ}$.

\section{Limitations}

This study utilised a relatively small student sample (31) whose members had some knowledge of gait analysis and training was not provided. Different results may have been obtained if analysts experienced in gait analysis or forensic gait analysis were used. For example, analysts experienced in using an oblique, front-side view may be more likely to incorporate the effect of this camera position into their judgement/interpretation, rather than merely reporting what they can observe. However, Birch et al. [4] compared the ability of both expert and novice observers to recognise walkers using visual gait analysis and reported no statistical significant difference. Whilst statistical differences in whole body gait patterns were not found, it is possible that subtle differences in features of gait (such as foot orientation) may be more easily identified by expert observers than novices.

This study used an avatar as the subject walker to control for potential confounding variables associated with the walker and their environment. Whilst this had a number of advantages, the obvious disadvantage is that this is a computer-generated model. Though the avatar is constructed using human movements from 3-dimensional motion capture [12], it does not cover all of the movements demonstrated by a human walker. Participants may be more accustomed to observing human walkers than computer generated models, which may require more practice. However, an advantage for research is that the majority of factors could be controlled and only the camera angle and foot orientations were altered to establish their effect on the scoring capacities of the observers.

The participants in this study were asked to comment on foot position during the stance phase of gait. However, participant factors could not be controlled. As such, it is also possible that participants used the foot position during the swing phase of gait. In human walking, the positions adopted by the foot during the swing and stance phases of gait can vary for one person but in this study, foot position was held constant in abduction for both of these phases. Therefore, the posterior view $\left(180^{\circ}\right)$ may have yielded more correct responses because participants used the foot position during the swing phase of gait to form their judgements.

A number of variables were fixed for consistency that may have influenced the outcomes observed in this research. For example, the camera position was fixed at 1.6 metres above the floor, a brick effect (with linear markings) was incorporated into the walking surface, the lighting and resolution were 
good and the avatar made at least 7 foot-ground contacts. In practice, the height of the camera above the ground can vary, the lighting, resolution and contrast can be poor and the figure of interest may only take a small number of steps or may demonstrate pathological gait. These additional factors in isolation, or in combination with each other and with viewing angle, may influence a practitioner's perception of angle of gait differently to when angle of view is the only manipulated variable. It was not within the scope of this study to test the effects of each variable in isolation and combination, therefore further research is recommended to explore their influence.

\section{Conclusion}

In this study, the overall level of accuracy exhibited by participants making judgements about angle of gait using various viewing angles in a controlled environment was very high (84.4\%). Where inaccurate judgements occurred, they could be attributed to changes in viewing angle and/or the foot position relative to the camera. The results of this study suggest that the most reliable viewing angle to make judgements about the angle of the feet was from the figure's back, in the frontal plane. The most unreliable viewing angle to make judgements about the angle of the feet was obliquely, from the figure's front and side. Erroneous judgements were particularly apparent in the foot furthest from the camera. The results suggest that viewing angle alone can influence judgements made about angle of gait, and practitioners should be mindful of this when analysing, comparing and evaluating this feature as part of forensic gait analysis. In practice, and in an uncontrolled environment, additional variables as described by Birch et al. [5] are likely to exist in CCTV footage. These factors may be present in various combinations and have the potential to positively or negatively influence observations made. Therefore, these results should not prevent forensic gait analysts from analysing angle of gait using oblique (front-side) viewing angles, but should be used to assist and guide their decision making process. Further research is required to establish whether viewing angle can affect judgements made about other features of gait to assist with our understanding of margin of uncertainty.

\section{References}


[1] The Forensic Science Regulator, Code of practice for forensic gait analysis, Issue 1, The Forensic Science Regulator, Birmingham, 2019, p. 4.

[2] The Royal Society and the Royal Society of Edinburgh, Forensic Gait Analysis: A Primer for Courts, The Royal Society, London, 2017.

[3] J.A. DiMaggio, D.W. Vernon, Forensic Podiatry: Principles and Methods, CRC Press, 2017

[4] I, Birch, M, Birch, N, Asgeirsdottir, The identification of individuals by observational gait analysis using closed circuit television footage, Science and Justice, 60 (2020) 79-85

[5] I. Birch, W. Vernon, J. Walker, J. Saxelby, The development of a tool for assessing the quality of closed circuit camera footage for use in forensic gait analysis, Journal of Forensic and Legal Medicine, 20 (2013) 915-917.

[6] Codes of practice and conduct for forensic science providers and practitioners in the Criminal Justice System. The Forensic Science Regulator, Birmingham, 2017.

[7] I. Birch, L. Raymond, A. Christou, M.A. Fernando, N. Harrison, F. Paul, The identification of individuals by observational gait analysis using closed circuit television footage, Science and Justice 53 (2013) 339-342.

[8] P. Larsen, E.B. Simonsen, N. Lynnerup, Gait Analysis in Forensic Medicine, Journal of Forensic Science, 53(5) (2008) 1149-1153

[9] Make Human Community, http://www.makehumancommunity.org, 2019 (accessed 09 March 2020)

[10] Blender, www.blender.org, 2019 (accessed 09 March 2020)

[11] I. Birch, W. Vernon, G. Burrow, J. Walker, The effect of frame rate on the ability of experienced gait analysts to identify characteristics of gait from closed circuit television footage, Science and Justice 54 (2014) 159-163.

[12] I. Birch, M. Birch, L. Rutler, S. Brown, L.R. Burgos, B. Otten, M. Wiedemeijer, The repeatability and reproducibility of the Sheffield features of gait tool, Science and Justice 59 (2019) 544-551.

[13] I, Birch, C. Gwinnett, J. Walker, Aiding the interpretation of forensic gait analysis: Development of a features of gait database, Science and Justice, 58 (2018) 78-82

[14] Z. Geradts, M. Merlijn, G, de Groot, J. Bijhold, Use of gait parameters of persons in video surveillance systems, Proceedings of SPIE, 4709 (2002) 16-24

[15] M. T. Cibulka, K. Winters, T. Kampwerth, B. McAfee, L. Payne, T. Roeckenhaus, S. A. Ross, Predicting foot progression angle during gait using two clinical measures in healthy adults, a preliminary study, International Journal of Sports Physical Therapy, 11(3), (2016) 400-408

[16] S. Seber, B. Hazer, N. Kose, E. Gokturk, I. Gunal, A. Turgut, Rotational profile of the lower extremity and foot progression angle: computerized tomographic examination of 50 male adults, Archives of orthopaedic and trauma surgery 120 (5-6) (2000) 255-258.

[17] S. Yu, D. Tan, T. Tan, A framework for evaluating the effect of view angle, clothing and carrying condition on gait recognition, Conference paper: $18^{\text {th }}$ International Conference on Pattern Recognition (2006), Hong Kong, China. DOI: 10.1109/ICPR.2006.67 
[18] D. Jokisch, I. Daum, N. F. Troje, Self-recognition versus recognition of others by biological motion: Viewpoint-dependent effects, Perception, 35 (2006) 911-920 